\title{
Understanding Superconductivity
}

The cold world of superconductivity celebrates some memorable anniversaries this year and next. Seventy-five years ago, in 1911, Dutch physicist Heike KamerlinghOnnes discovered that some metals lost all electrical resistance when cooled to nearly absolute zero.

Called superconductivity, this low-temperature property came as a bit of a shock to many scientists at the time. Lord Kelvin had predicted in 1902 that as a metal was cooled its resistance should reach a minimum but then rise to become infinite as the electrons eventually condensed onto the metal atoms.

A leading low-temperature experimenter, Kamerlingh-Onnes founded the notable Cryogenics Laboratory in Leiden, first liquified helium (in 1908), and even coined the word cryogen (from the Greek kryos for cold and -gen meaning to become.) Before his experiments, Kamerlingh-Onnes had thought the resistance of cold metals would go to zero because the source of the resistance, Planck vibrators, would lose their energy at the lowest temperatures.

But, in the words of Leiden experimentalist Hendrik B.G. Casimir, "If the atomic motion would follow the laws of classical mechanics, low-temperature physics might well be a dull subject." Which, of course, was not to be. On the surface, though, superconductivity might look dull even to an enthusiast.

Casimir recalled that he and his wife Josina "in youthful enthusiasm, once observed a whole night long ... the current circulating in a circuit."

"We did not find any change in the current," he said. "I like to say that the resistance of superconductors is about the zeroest quantity we know."

But at an atomic level, a lot was going on to create that zero condition.

Kamerlingh-Onnes found that the metals showed a discontinuous decrease of resistance to zero and that different metals had different transition temperatures. This meant a new explanation of superconductivity was needed.

Not until 1957, 30 years ago next year, was a comprehensive theory devised to describe superconductivity. This is the famous BCS theory, which won its originators-John Bardeen, Leon Cooper, and J. Robert Shrieffer - the 1972 Nobel Prize for Physics. The trio found that the key to understanding superconductivity is a pairing interaction between the electrons resulting from an effective at traction induced by the interaction between electrons and atomic vibrations.

The BCS theory not only accounted for the major aspects of superconductivity, but was used to predict new phenomena and gave a great stimulus to the field.

Foremost of the new phenomena were those concerning two strips of superconductor separated by a thin oxide predicted by Brian D. Josephson a quarter century ago (1961-1962) when he was a research student at the Mond Laboratory in Cambridge (England).

Josephson calculated that two supercurrents would form in the type of "junction" that now bears his name. One current would be continuous direct current, persisting without an electromotive force just as it would in an ordinary superconductor. The second current, which appears when a voltage difference is established between the two superconductor strips, is an alternating current with a frequency independent of materials used in the junction.

The Josephson currents resulted from quantum mechanical "tunneling" phenomena, in which electrons pass back and forth through a region (the oxide) where they had zero probability of ever being.

"For an engineer, it sounds rather strange that if you throw a tennis ball against a wall enough times it will eventually go through without damaging either the wall or it self," said Ivan Giaever, a General Electric engi- neer turned experimental physicist. "The trick, of course, is to use very tiny balls, and lots of them."

Giaever had seen the Josephson effect many times in his extensive tunneling experiments. But he had discarded those junctions as having electrical shorts between the superconductors.

"Later I have been asked many times if I feel bad for missing the effect," Giaever said. "The answer is clearly no, because to make an experimental discovery it is not enough to observe something. One must also realize the significance of the observation, and in this instance I was not even close."

Giaever's remarks followed his acceptance of the 1973 Nobel Prize for Physics, which he shared with Josephson and Leo Esaki of IBM, the inventor of the tunnel diode.

After making his initial calculations, Josephson was said to be a bit uneasy that they showed the current to be dependent on the phase difference in the superconductors. Philip W. Anderson, an influential instructor of Josephson's that year in Cambridge, said later that the uncertainty of Josephson and his thesis advisor Brian Pippard may have led them to send the landmark paper to a new journal, Physics Letters, rather than to the established Physical Review Letters.

In time, the Josephson phenomena were found to be so reliable and precise they have been used to define the unit of voltage, the atomic constant $e / h$, and to make extremely sensitive detecting devices for currents, voltages, and magnetic fields. While individual circuits have been made, however, the promise of exceedingly fast computers using Josephson junction-based integrated circuits has not yet been realized commercially.

M. ROSS

\section{It's time to renew your MRS membership!}

\author{
Don't miss the BULLEIIN or any of your membership benefits-subscription to Journal \\ of Materials Research, advance details of MRS meetings, discounts on MRS \\ proceedings and other publications, and more
}

Look for your membership renewal notice in the mail. 